\title{
Optically induced topological states on the surface of mercury telluride
}

\author{
O. Kyriienko, ${ }^{1,2}$ O. V. Kibis, ${ }^{3,4}$, , and I. A. Shelykh ${ }^{2,4}$ \\ ${ }^{1}$ NORDITA, KTH Royal Institute of Technology and Stockholm University, \\ Roslagstullsbacken 23, SE-106 91 Stockholm, Sweden \\ ${ }^{2}$ ITMO University, St. Petersburg 197101, Russia \\ ${ }^{3}$ Department of Applied and Theoretical Physics, Novosibirsk State Technical University, \\ Karl Marx Avenue 20, 630073 Novosibirsk, Russia \\ ${ }^{4}$ Science Institute, University of Iceland IS-10\%, Reykjavik, Iceland
}

\begin{abstract}
We developed the theory which describes the Floquet engineering of surface electronic modes in bulk mercury telluride ( $\mathrm{HgTe}$ ) by a circularly polarized electromagnetic field. The analysis shows that the field results in appearance of the surface states which arise from the mixing of conduction and valence bands of HgTe. Their branches lie near the center of the Brillouin zone and have the Dirac dispersion characteristic for topological states. Besides them, the irradiation induces the gap between the conduction and valence bands of $\mathrm{HgTe}$. Thus, the irradiation can turn mercury telluride into topological insulator from gapless semiconductor. It is demonstrated that the optically induced states differ substantially from the non-topological surface states existing in $\mathrm{HgTe}$ without irradiation. The structure of the found states is studied both analytically and numerically in the broad range of their parameters.
\end{abstract}

\section{INTRODUCTION}

In the last years, the achievements in the laser and microwave techniques have made possible the optical control of condensed matter structures with a highfrequency electromagnetic field (so-called dressing field), which is based on the Floquet theory of periodically driven quantum systems (Floquet engineering) $\underline{\underline{1}} \underline{\underline{\underline{4}}}$. Particularly, the studies of various nanostructures strongly coupled to light - including quantum wells ${ }^{5-9}$, quantum rings $\underline{10}-13$, quantum spin chains $\stackrel{14}{-17}$, graphene and Dirac materials $18-27$ etc. - have emerged as a vibrant area of contemporary physics with the objective to control electronic properties of these systems.

Among various low-dimensional electronic systems, electrons localized near boundaries of condensed matter structures (surface electronic states) bear a special role. The increasing interest of scientific community devoted to them is caused by the topologically nontrivial nature of the surface states in structures known as topological insulators $\frac{28-30}{-3}$ which behave like an insulator in their bulk but have the gapless conducting electronic modes protected by the time-reversal symmetry at their boundaries. As a consequence, the Floquet engineering of topological surface states (Floquet topological insulators) attracts attention as an effective tool to control their physical properties. Particularly, it is shown that the irradiation with a high-frequency electromagnetic field induces topological edge states in graphene $\underline{31.32}$ and semiconductor quantum wells 33,34 . Optically induced Weyl points have been predicted in topological insulators and Dirac semimetals $35-39$. Recently, the theory of opticallycontrolled spin transport on the surface of bulk topological insulators was elaborated ${ }^{40}$, light-induced modification of surface topological states in thin films was studied $\stackrel{41}{ }$, and the optically induced topological edge states in the array of quantum rings were analyzed $\underline{42}$.

In the present article, we apply the Floquet engineering approach to the bulk gapless semiconductor-mercury telluride $(\mathrm{HgTe})$. Although this material has shown topologically nontrivial electronic properties actively studied last years, only strain was considered before to turn bulk $\mathrm{HgTe}$ into topological insulator ${ }^{43} \underline{-50}$. In contrast to this, we demonstrate theoretically that a circularly polarized high-frequency electromagnetic field can create topological electronic states on HgTe surface. Since the same field opens the gap between conduction and valence bands, the light-induced topological phase transition (which turns $\mathrm{HgTe}$ from gapless semiconductor into topological insulator) occurs.

The article is organized as follows. In Sec. II, we formulate the Hamiltonian formalism describing the electronic states on the irradiated surface of HgTe. In Sec. III, we solve the corresponding Schrödinger problem analytically in the simplest particular cases, calculate the dispersion of the surface states numerically, and analyze their energy spectrum. This is followed by the conclusion.

\section{THE HAMILTONIAN}

In the present article, we consider the surface electronic states which are localized near the surface (001) of bulk $\mathrm{HgTe}$ and originate from the light-induced mixing of conduction and valence bands near the center of the Brillouin zone. First of all, let us write the Hamiltonian describing these bands in bulk $\mathrm{HgTe}^{51}$ without a dressing field,

$$
\hat{\mathcal{H}}=\hat{\mathcal{H}}_{\mathrm{L}}+\hat{\mathcal{H}}_{\mathrm{BIA}},
$$

where

$$
\begin{aligned}
\hat{\mathcal{H}}_{\mathrm{L}} & =\left(\gamma_{1}+5 \gamma_{2} / 2\right) \mathbf{k}^{2}-2 \gamma_{2}\left(J_{x}^{2} k_{x}^{2}+J_{y}^{2} k_{y}^{2}+J_{z}^{2} k_{z}^{2}\right) \\
& -2 \gamma_{3}\left(\left\{J_{x}, J_{y}\right\} k_{x} k_{y}+\left\{J_{x}, J_{z}\right\} k_{x} k_{z}+\left\{J_{y}, J_{z}\right\} k_{y} k_{z}\right)
\end{aligned}
$$



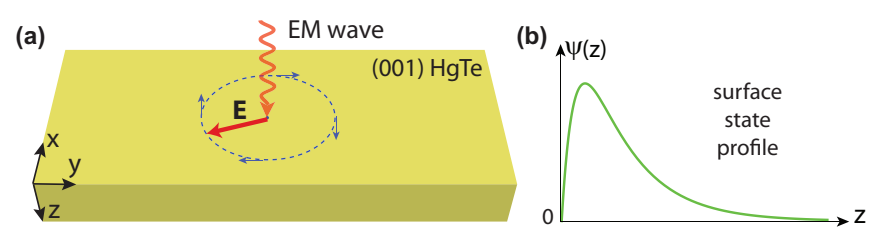

FIG. 1: Sketch of the system under consideration: (a) (001) surface of bulk HgTe, irradiated by a circularly polarized electromagnetic wave with the electric field amplitude $\mathbf{E}$; (b) wave function, $\Psi(z)$, of surface electronic states localized near the (001) surface of $\mathrm{HgTe}$.

is the Luttinger Hamiltonian,

$$
\begin{aligned}
\hat{\mathcal{H}}_{\mathrm{BIA}} & =\alpha\left[k_{x}\left\{J_{x},\left(J_{y}^{2}-J_{z}^{2}\right)\right\}+k_{y}\left\{J_{y},\left(J_{z}^{2}-J_{x}^{2}\right)\right\}\right. \\
& \left.+k_{z}\left\{J_{z},\left(J_{x}^{2}-J_{y}^{2}\right)\right\}\right]
\end{aligned}
$$

is the term coming from the bulk inversion asymmetry (BIA) of the crystal structure, $\mathbf{k}=\left(k_{x}, k_{y}, k_{z}\right)$ is the electron wave vector, $\gamma_{1,2,3}$ are the Luttinger parameters, $\alpha$ is the BIA parameter, $J_{x, y, z}$ are the $4 \times 4$ matrices corresponding to the electron angular momentum $J=3 / 2$, and the curly brackets $\{A, B\}$ represent the anti-commutators of the matrices $A$ and $B$. To perform calculations, it is convenient to rewrite the Hamiltonian (11) as a $4 \times 4$ matrix in the basis of Luttinger-Kohn wave functions, $\psi_{j_{z}}$, which describe four-fold degenerate electron states of the conduction and valence band in the center of the bulk Brillouin zone, and correspond to the four different projections of electron momentum on the $z$ axis, $j_{z}= \pm 1 / 2$ and $j_{z}= \pm 3 / 2$ (see e.g. Ref. [51]). In this basis, the Hamiltonian (11) reads

$$
\hat{\mathcal{H}}=\begin{array}{|c||cccc|}
\hline j_{z} \backslash j_{z} & +3 / 2 & -1 / 2 & +1 / 2 & -3 / 2 \\
\hline \hline+3 / 2 & F & I+L & H+M & N \\
-1 / 2 & I^{*}+L & G & -N & -H+M \\
+1 / 2 & H^{*}+M^{*} & -N^{*} & G & I-L \\
-3 / 2 & N^{*} & -H^{*}+M^{*} & I^{*}-L & F \\
\hline
\end{array}
$$

where the matrix elements are

$$
\begin{aligned}
& F=\left(\gamma_{1}+\gamma_{2}\right)\left(k_{x}^{2}+k_{y}^{2}\right)+\left(\gamma_{1}-2 \gamma_{2}\right) k_{z}^{2}, \\
& G=\left(\gamma_{1}-\gamma_{2}\right)\left(k_{x}^{2}+k_{y}^{2}\right)+\left(\gamma_{1}+2 \gamma_{2}\right) k_{z}^{2}, \\
& I=-\sqrt{3} \gamma_{2}\left(k_{x}^{2}-k_{y}^{2}\right)+i 2 \sqrt{3} \gamma_{3} k_{x} k_{y}, L=\sqrt{3} \alpha k_{z}, \\
& M=-(\sqrt{3} \alpha / 2)\left(k_{x}+i k_{y}\right), H=-2 \sqrt{3} \gamma_{3}\left(k_{x}-i k_{y}\right) k_{z}, \\
& N=-(3 \alpha / 2)\left(k_{x}-i k_{y}\right),
\end{aligned}
$$

Next, we add a dressing field which introduces the optically induced mixing of conduction and valence bands. Within the conventional minimal coupling approach, we perform the replacement $\mathbf{k} \rightarrow e \mathbf{A}(t) / \hbar$ in the Hamiltonian (11), where $\mathbf{A}=\left(A_{x}, A_{y}, A_{z}\right)$ is the time-dependent vector potential of the dressing field near the irradiated surface. Assuming the electromagnetic wave (dressing field) to be circularly polarized and propagating along the $z$ axis (see Fig. 17), the vector potential near the irradiated (001) surface of $\mathrm{HgTe}$ can be written as

$$
\mathbf{A}=\frac{E}{\omega}(\cos \omega t, \sin \omega t, 0),
$$

where $E$ and $\omega$ are the amplitude and frequency of the EM wave, respectively. Then the Hamiltonian (1) can be rewritten as

$$
\hat{\mathcal{H}}(t)=\hat{\mathcal{H}}_{0}+\left[\hat{V}_{1} e^{i \omega t}+\hat{V}_{2} e^{i 2 \omega t}+\text { H.c. }\right],
$$

where the time-independent part is

$$
\hat{\mathcal{H}}_{0}=\hat{\mathcal{H}}_{\mathrm{L}}+\hat{\mathcal{H}}_{\mathrm{BIA}}+\left(\gamma_{1}+5 \gamma_{2} / 2\right) k_{0}^{2}-\gamma_{2}\left(J_{x}^{2}+J_{y}^{2}\right) k_{0}^{2},
$$

and the two harmonics are

$$
\begin{aligned}
\hat{V}_{1} & =\left[\left(\gamma_{1}+5 \gamma_{2} / 2\right)\left(k_{x}-i k_{y}\right)-2 \gamma_{2}\left(J_{x}^{2} k_{x}-i J_{y}^{2} k_{y}\right)\right. \\
& -\gamma_{3}\left(\left\{J_{x}, J_{y}\right\}\left(k_{y}-i k_{x}\right)+\left\{J_{x}-i J_{y}, J_{z}\right\} k_{z}\right) \\
& \left.+(\alpha / 2)\left(\left\{J_{x},\left(J_{y}^{2}-J_{z}^{2}\right)\right\}-i\left\{J_{y},\left(J_{z}^{2}-J_{x}^{2}\right)\right\}\right)\right] k_{0} \\
\hat{V}_{2} & =\left[\left(\gamma_{2} / 2\right)\left(J_{y}^{2}-J_{x}^{2}\right)+i\left(\gamma_{3} / 2\right)\left\{J_{x}, J_{y}\right\}\right] k_{0}^{2}
\end{aligned}
$$

Here, $k_{0}=|e| E / \hbar \omega$ is the amplitude of the field-induced shift of the in-plane electron wave vector. Applying the conventional Floquet-Magnus approach $\underline{52} \underline{-54}$ to renormalize the time-dependent Hamiltonian (6), we arrive at the effective time-independent Hamiltonian,

$$
\begin{aligned}
& \hat{\mathcal{H}}_{\text {eff }}=\hat{\mathcal{H}}_{0}+\frac{\left[\hat{V}_{1}, \hat{V}_{1}^{\dagger}\right]}{\hbar \omega}+\frac{\left[\hat{V}_{2}, \hat{V}_{2}^{\dagger}\right]}{2 \hbar \omega} \\
& +\frac{\left[\left[\hat{V}_{1}, \hat{\mathcal{H}}_{0}\right], \hat{V}_{1}^{\dagger}\right]+\text { H.c. }}{2(\hbar \omega)^{2}}+\frac{\left[\left[\hat{V}_{2}, \hat{\mathcal{H}}_{0}\right], \hat{V}_{2}^{\dagger}\right]+\text { H.c. }}{8(\hbar \omega)^{2}} \\
& +o\left(\frac{\hat{V}_{1,2}}{\hbar \omega}\right)^{2}
\end{aligned}
$$

where the square brackets $[\hat{A}, \hat{B}]$ represent the commutators of the operators $\hat{A}$ and $\hat{B}$. In what follows, we consider the dressing field (5) to be high-frequency $\left(\gamma_{1,2,3} k_{0}^{2} / \hbar \omega \ll 1\right.$ and $\left.\alpha k_{0} / \hbar \omega \ll 1\right)$. For reasonable irradiation intensities of $\mathrm{kW} / \mathrm{cm}^{2}$ scale, these conditions can be satisfied for high-frequencies beginning from the $\mathrm{THz}$ range. In this high-frequency limit, the effective timeindependent Hamiltonian (91) reads $\hat{\mathcal{H}}_{\text {eff }} \approx \hat{\mathcal{H}}_{0}$. Therefore, one can use the stationary Hamiltonian (7) to describe the electronic states near the (001) surface renormalized by the high-frequency field (5). In the LuttingerKohn basis, $\psi_{j_{z}}$, the Hamiltonian (7) reads

$$
\hat{\mathcal{H}}_{0}=\begin{array}{|c||cccc|}
\hline j_{z} \backslash j_{z} & +3 / 2 & -1 / 2 & +1 / 2 & -3 / 2 \\
\hline \hline+3 / 2 & F_{0} & I+L & H+M & N \\
-1 / 2 & I^{*}+L & G_{0} & -N & -H+M \\
+1 / 2 & H^{*}+M^{*} & -N^{*} & G_{0} & I-L \\
-3 / 2 & N^{*} & -H^{*}+M^{*} & I^{*}-L & F_{0} \\
\hline
\end{array}
$$


where the field-containing matrix elements are $F_{0}=F+$ $\Delta_{0}+\Delta / 2$ and $G_{0}=G+\Delta_{0}-\Delta / 2, \Delta=2 \gamma_{2}(e E / \hbar \omega)^{2}$ is the field-induced splitting of the conduction and valence bands in the Brillouin zone center, and $\Delta_{0}=\gamma_{1}(e E / \hbar \omega)^{2}$ is the field-induced shift of zero energy, which is omitted in the following.

\section{RESULTS AND DISCUSSION}

First of all, let us consider the electronic states with the zero in-plane wave vector, $k_{x}=k_{y}=0$. For these states, the Floquet engineered Hamiltonian (10) takes the block-diagonal form

$$
\hat{\mathcal{H}}_{0}=\left[\begin{array}{cc}
\hat{\mathcal{H}}^{ \pm} & 0 \\
0 & \hat{\mathcal{H}}^{\mp}
\end{array}\right]
$$

where

$$
\hat{\mathcal{H}}^{ \pm}=\left[\begin{array}{cc}
\left(\gamma_{1} \mp 2 \gamma_{2}\right) k_{z}^{2} \pm \Delta / 2 & \pm \sqrt{3} \alpha k_{z} \\
\pm \sqrt{3} \alpha k_{z} & \left(\gamma_{1} \pm 2 \gamma_{2}\right) k_{z}^{2} \mp \Delta / 2
\end{array}\right] .
$$

The four eigenspinors of the Hamiltonian (11) describing bulk electronic states of $\mathrm{HgTe}$ at $k_{x}=k_{y}=0$ can be written as

$$
\varphi_{j}^{+}=\left[\begin{array}{c}
\lambda_{j}^{+}\left(k_{z}\right) \\
1 \\
0 \\
0
\end{array}\right] e^{i k_{z} z}, \varphi_{j}^{-}=\left[\begin{array}{c}
0 \\
0 \\
1 \\
\lambda_{j}^{-}\left(k_{z}\right)
\end{array}\right] e^{i k_{z} z}, j=1,2,
$$

where

$$
\lambda_{j}^{ \pm}\left(k_{z}\right)=\frac{\mp \sqrt{3} \alpha k_{z}}{\left(\gamma_{1} \mp 2 \gamma_{2}\right) k_{z}^{2} \pm \Delta / 2-\varepsilon_{j}\left(k_{z}\right)},
$$

and the corresponding eigenenergies are

$$
\varepsilon_{j}\left(k_{z}\right)=\gamma_{1} k_{z}^{2}+(-1)^{j} \sqrt{\left(\Delta / 2-2 \gamma_{2} k_{z}^{2}\right)^{2}+3 \alpha^{2} k_{z}^{2}} .
$$

The electronic states localized near the surface (001) are described by the same spinors (13) with the imaginary $z$-component of electron wave vector, $k_{z}=i \kappa$. The two energy branches (15) produce two different parameters $\kappa=\kappa_{1,2}$ satisfying the equation

$$
\varepsilon=\gamma_{1} \kappa_{j}^{2}+(-1)^{j} \sqrt{\left(\Delta / 2+2 \gamma_{2} \kappa_{j}^{2}\right)^{2}-3 \alpha^{2} \kappa_{j}^{2}},
$$

where $\varepsilon$ is the energy of the surface state. It should be stressed that the parameters $\kappa_{1,2}$ can be complex numbers but their real part must be positive for spinors (13) to decay exponentially into the bulk at $z \rightarrow \infty$ (see Fig. 10). Making the replacement, $k_{z} \rightarrow i \kappa_{1,2}$, in Eqs. (13)-(15), one can write the surface-localized eigenfunction of the Hamiltonian (11) as a linear combination of the spinors (13),

$$
\begin{aligned}
& \Psi(z)=C_{1}\left[\begin{array}{c}
\lambda_{1}^{+}\left(i \kappa_{1}\right) \\
1 \\
0 \\
0
\end{array}\right] e^{-\kappa_{1} z}+C_{2}\left[\begin{array}{c}
\lambda_{2}^{+}\left(i \kappa_{2}\right) \\
1 \\
0 \\
0
\end{array}\right] e^{-\kappa_{2} z} \\
& +C_{3}\left[\begin{array}{c}
0 \\
0 \\
1 \\
\lambda_{1}^{-}\left(i \kappa_{1}\right)
\end{array}\right] e^{-\kappa_{1} z}+C_{4}\left[\begin{array}{c}
0 \\
0 \\
1 \\
\lambda_{2}^{-}\left(i \kappa_{2}\right)
\end{array}\right] e^{-\kappa_{2} z}
\end{aligned}
$$

where $C_{1,2,3,4}$ are the constants to be determined. To do so, we chose the model of a surface potential which can be approximated by the infinitely-high barrier at the coordinate $z=0$. This sets the boundary condition for the electron wave function (17) as $\left.\Psi\right|_{z=0}=0$, and results into a homogeneous system of four algebraic equations defining the constants $C_{1,2,3,4}$,

$$
\begin{aligned}
& C_{1} \lambda_{1}^{+}\left(i \kappa_{1}\right)+C_{2} \lambda_{2}^{+}\left(i \kappa_{2}\right)=0, C_{1}+C_{2}=0, \\
& C_{3} \lambda_{1}^{-}\left(i \kappa_{1}\right)+C_{4} \lambda_{2}^{-}\left(i \kappa_{2}\right)=0, C_{3}+C_{4}=0 \text {. }
\end{aligned}
$$

The secular equation for the algebraic system (18),

$$
\left[\lambda_{1}^{+}\left(i \kappa_{1}\right)-\lambda_{2}^{+}\left(i \kappa_{2}\right]\left[\lambda_{1}^{-}\left(i \kappa_{1}\right)-\lambda_{2}^{-}\left(i \kappa_{2}\right)\right]=0,\right.
$$

defines the sought energy (16) of the surface electronic states at $k_{x}=k_{y}=0$.

The system of equations (19) and (16) can be easily solved analytically if $\gamma_{1}=0$. Physically, this particular case corresponds to a semiconductor with the Hamiltonian (2), where the masses of electrons and holes along the $z$ axis, $m_{e}$ and $m_{h}$, are equal to each other. For such a symmetric electron-hole system, the eigenenergy (16) is $\varepsilon=0$ and the surface-localized eigenspinors (17) corresponding to this eigenenergy can be written as

$$
\begin{aligned}
& \Psi_{1}(z)=A\left(e^{-\kappa_{1} z}-e^{-\kappa_{2} z}\right)\left[\begin{array}{c}
-i \\
1 \\
0 \\
0
\end{array}\right], \\
& \Psi_{2}(z)=A\left(e^{-\kappa_{1} z}-e^{-\kappa_{2} z}\right)\left[\begin{array}{l}
0 \\
0 \\
1 \\
i
\end{array}\right],
\end{aligned}
$$

where

$$
\kappa_{j}=\left|\frac{\sqrt{3} \alpha}{4 \gamma_{2}}\right|+(-1)^{j} \sqrt{\left(\frac{\sqrt{3} \alpha}{4 \gamma_{2}}\right)^{2}-\frac{\Delta}{4 \gamma_{2}}},
$$

and $A=\sqrt{\sqrt{3} \alpha \Delta /\left(6 \alpha^{2}-8 \gamma_{2} \Delta\right)}$ is the normalization constant. The eigenspinors (20)-(21) can be easily verified by direct substitution into the Schrödinger equation, $\hat{\mathcal{H}} \Psi_{1,2}=\varepsilon \Psi_{1,2}$, with the Hamiltonian (11) and the eigenenergy $\varepsilon=0$. Since $\kappa_{1,2} \neq 0$ if $\alpha \neq 0$, the surface states (20)-(21) physically originate from the BIA of 
the crystal. To find the dispersion of the surface states (20)-(21) for small in-plane wave vectors $k_{x, y}$, we have to project the total Hamiltonian (10) to the subspace spanned by these two states, $\Psi_{1}$ and $\Psi_{2}$. Keeping the terms linear in $k_{x, y}$, we arrive at the Hamiltonian,

$$
\hat{\mathcal{H}}_{\mathrm{D}}=-\frac{3 \alpha}{2}\left(\sigma_{x} k_{x}+\sigma_{y} k_{y}\right)-\frac{\sqrt{3} \alpha}{2}\left(\sigma_{x} k_{y}+\sigma_{y} k_{x}\right),
$$

where $\sigma_{x, y}$ are the Pauli matrices written in the basis (20)-(21). Diagonalizing the Hamiltonian (23), we can write the sought energy spectrum of the surface states (201)-(21) near $k_{x}=k_{y}=0$ as

$$
\varepsilon\left(k_{x}, k_{y}\right)= \pm \sqrt{3} \alpha \sqrt{k_{x}^{2}+k_{y}^{2}+\sqrt{3} k_{x} k_{y}} .
$$

Eqs. (23)-(24) reveal the energy spectrum of the found surface states, which is typical for topological insulators ${ }^{28}$. Namely, the two degenerate states (20)-(21) form the Dirac point at $k_{x}=k_{y}=0$ with the energy $\varepsilon=0$, which lies in the middle of the conduction and valence bands, and the linear dispersion (24) near the point appears.
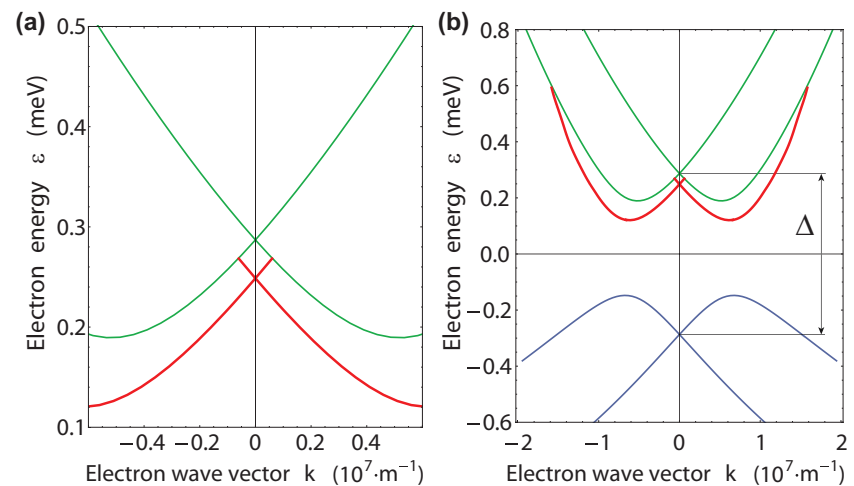

FIG. 2: The energy spectrum of the optically induced topological electronic states (bold red curves) on the (001) surface of $\mathrm{HgTe}$ irradiated by a circularly polarized electromagnetic wave with the intensity $I=850 \mathrm{~W} / \mathrm{cm}^{2}$ and the photon energy $\hbar \omega=2 \mathrm{meV}$ : (a) near the Dirac point; (b) in the broad range of electron wave vectors. Thin green and blue curves represent the dispersion of conduction and valence bands in bulk $\mathrm{HgTe}$, respectively, and $\Delta$ is the optically induced band gap.

The approach discussed above, which describes analytically the surface states originated from the Hamiltonian (10) at $k_{x}=k_{y}=0$ and $\gamma_{1}=0$, can be applied numerically to the same Hamiltonian (10) at any wave vectors $k_{x, y}$ and parameters $\gamma_{1,2,3}$ as well. As a result, one can calculate the energy spectrum of the surface states in the broad range of electron wave vectors and band parameters. The numerically calculated dispersion of the surface states is presented in Fig. 2 for the following $\mathrm{HgTe}$ band parameters ${ }^{50.55}: \gamma_{1}=15.6 \hbar^{2} / 2 m_{0}$, $\gamma_{2}=9.6 \hbar^{2} / 2 m_{0}, \gamma_{3}=8.6 \hbar^{2} / 2 m_{0}$ and $\alpha=0.208 \AA \cdot \mathrm{eV}$. Since the electron-hole system in HgTe is strongly asymmetric, $m_{e} / m_{h}=\left(\gamma_{1}-2 \gamma_{2}\right) /\left(\gamma_{1}+2 \gamma_{2}\right) \ll 1$, the Dirac point energy is shifted towards the conduction band (see Fig. 2a). It is seen also that the branches of the surface states merge into the spectrum of bulk conduction band if the plane electron wave vector, $k_{x, y}$, is large enough (see Fig. 2b). As a result, the discussed surface states as a whole are localized near the conduction band of $\mathrm{HgTe}$ for small wave vectors $k$. It should be stressed that the Hamiltonian (23) and the dispersion (24) are applicable to describe the energy spectrum of surface states near the Dirac point for any band parameters. Particularly, the Dirac velocity, $v_{D}=\sqrt{3} \alpha / \hbar$, which can be extracted from the dispersion (24), does not depend on the Luttinger parameters $\gamma_{1,2,3}$. We note that the effective Hamiltonian (10) is similar to the Hamiltonian of a strained gapless semiconductor $\underline{51}$. Therefore, the discussed topological states behave like those in strained $\mathrm{HgTe}^{\underline{50}}$.
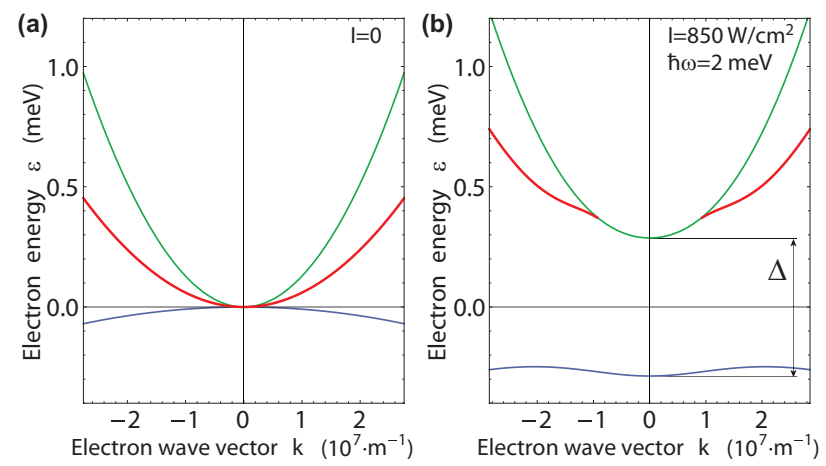

FIG. 3: The energy spectrum of the D'yakonov-Khaetskii surface electronic states (bold red curves) on the (001) surface of HgTe:(a) in the absence of irradiation; (b) in the presence of a circularly polarized electromagnetic wave with the intensity $I=850 \mathrm{~W} / \mathrm{cm}^{2}$ and the photon energy $\hbar \omega=2 \mathrm{meV}$. Thin green and blue curves represent the dispersion of bulk bands, respectively, and $\Delta$ is the optically induced band gap.

It follows from Eq. (22) that $\kappa_{1} \neq 0$ if the optically induced band gap is $\Delta \neq 0$. Otherwise, $\kappa_{1}=0$ and the corresponding electronic wave function plotted in Fig. 1b is delocalized, i.e. the surface states vanish. Therefore, the surface electronic states discussed above exist only in the presence of the irradiation and, thus, are optically induced. This is the substantial difference of the considered surface states from the non-topological surface states existing in gapless $\mathrm{HgTe}$ without an irradiation, which were analyzed for the first time by D'yakonov and Khaetskii ${ }^{56}$. To demonstrate the difference between these two kinds of surface states in more details, let us consider the evolution of the D'yakonov-Khaetskii (DKh) surface states under the irradiation. In contrast to the considered optically induced surface states, the BIA Hamiltonian (3) is not crucial for their existence. Therefore, we will assume $\alpha=0$ in the following analysis. To simplify calculations, we also neglect the weak anisotropy of electron-hole dispersion in HgTe. Mathematically, this corresponds to the replacement of the Luttinger param- 
eters, $\gamma_{2,3} \rightarrow \gamma=\left(2 \gamma_{2}+3 \gamma_{3}\right) / 5$, in the Hamiltonian (10). Under the made assumptions, the surface-localized eigenspinor of the Hamiltonian (10) can be written as

$$
\begin{gathered}
\Psi(z)=C_{1}\left[\begin{array}{c}
e^{-\frac{i 3 \theta}{2}} \\
\lambda_{1}^{+} e^{\frac{i \theta}{2}} \\
-i \lambda_{1}^{+} e^{-\frac{i \theta}{2}} \\
-i e^{\frac{i 3 \theta}{2}}
\end{array}\right] e^{-\kappa_{1} z}+C_{2}\left[\begin{array}{c}
e^{-\frac{i 3 \theta}{2}} \\
\lambda_{1}^{-} e^{\frac{i \theta}{2}} \\
i \lambda_{1}^{-} e^{-\frac{i \theta}{2}} \\
i e^{\frac{i 3 \theta}{2}}
\end{array}\right] e^{-\kappa_{1} z} \\
+C_{3}\left[\begin{array}{c}
i \lambda_{2}^{+} e^{-\frac{i 3 \theta}{2}} \\
-i e^{\frac{i \theta}{2}} \\
e^{-\frac{i \theta}{2}} \\
-\lambda_{2}^{+} e^{\frac{i 3 \theta}{2}}
\end{array}\right] e^{-\kappa_{2} z}+C_{4}\left[\begin{array}{c}
-i \lambda_{2}^{-} e^{-\frac{i 3 \theta}{2}} \\
i e^{\frac{i \theta}{2}} \\
e^{-\frac{i \theta}{2}} \\
-\lambda_{2}^{-} e^{\frac{i 3 \theta}{2}}
\end{array}\right] e^{-\kappa_{2} z},
\end{gathered}
$$

where

$$
\lambda_{j}^{ \pm}=\frac{(-1)^{j}\left[\varepsilon-\gamma_{1}\left(k^{2}-\kappa_{j}^{2}\right)\right]+\gamma\left(k^{2}+2 \kappa_{j}^{2}\right)+\Delta / 2}{\sqrt{3} \gamma\left(k^{2} \pm 2 \kappa_{j} k\right)},
$$

$\mathbf{k}=\left(k_{x}, k_{y}, 0\right)=(k \cos \theta, k \sin \theta, 0)$ is the in-plane wave vector, and the energy of the surface electronic states is

$$
\begin{aligned}
& \varepsilon=\gamma_{1}\left(k^{2}-\kappa_{j}^{2}\right) \\
& +(-1)^{j} \gamma \sqrt{3 k^{4}-12 \kappa_{j}^{2} k^{2}+\left[k^{2}+2 \kappa_{j}^{2}+\Delta /(2 \gamma)\right]^{2}} .
\end{aligned}
$$

Applying the zero boundary condition, $\Psi(0)=0$, to the eigenspinor (25), we arrive at the homogeneous system of four algebraic equations defining the constants $C_{1,2,3,4}$. The secular equation of the system reads as

$$
\left[\lambda_{1}^{+} \lambda_{2}^{-}+1\right]\left[\lambda_{1}^{-} \lambda_{2}^{+}+1\right]=0
$$

Solving this secular equation, one can find the energy spectrum of the DKh surface states in irradiated HgTe, $\varepsilon$. In the absence of the irradiation $(\Delta=0)$, Eq. (28) can be solved analytically and leads to the known dispersion of the DKh surface states 56 ,

$$
\varepsilon=\left[1-\left(\frac{1+\sqrt{3\left(2 \gamma-\gamma_{1}\right) /\left(2 \gamma+\gamma_{1}\right)}}{2}\right)^{2}\right]\left(\gamma_{1}+2 \gamma\right) k^{2}
$$

which is plotted in Fig. 3a. Since the DKh surface states are not topological, their dispersion is parabolic in contrast to the Dirac dispersion of the optically induced states plotted in Fig. 2. Solving Eq. (28) numerically for $\Delta \neq 0$, we arrive at the spectrum of the DKh states on the irradiated surface, which is shown in Fig. 3b. We observe that the discussed states exist only for large electron wave vectors, $k$, and vanish near $k=0$. Namely, it follows from Fig. 3 b that the branch of the DKh surface states merges into the continuum of bulk conduction band at a some critical electron wave vector. The value of the critical wave vector, $k=k^{\prime}$, is defined by Eq. (28), where the energy of the surface electron states (27) is equal to the energy of bulk conduction band in irradiated HgTe. Taking this into account, one can find $k^{\prime} \propto \sqrt{\Delta}$. Thus, the irradiation shifts the existence domain of the DKh states to the region of large electron wave vectors, $k$, inside the conduction band. As a consequence, they disappear near the Dirac point of the optically induced topological states shown in Fig. 2a. It should be noted also that the DKh states do not lie within the band gap $\Delta$ (see Fig.3b). Therefore, they cannot turn mercury telluride into topological insulator.

\section{CONCLUSION}

We developed the theory which describe surface electronic states appearing on the surface of $\mathrm{HgTe}$ due to the mixing of the conduction and valence bands by a circularly polarized electromagnetic field. The states originate from the bulk inversion asymmetry of $\mathrm{HgTe}$ and have the Dirac point in their dispersion, which is characteristic for topological states. It is shown that the structure of these optically induced topological states differs substantially from the known non-topological D'yakonovKhaetskii surface states 56 existing in $\mathrm{HgTe}$ in the absence of irradiation. Namely, the irradiation shifts these surface states to the region of large electron wave vectors, whereas the optically induced topological states are localized near the Brillouin zone center. As a consequence, these two kinds of surface states can be detected in experiments independently. It should be noted that the experimental methodology based on the angle resolved photoemission spectroscopy (ARPES) technique, which is commonly used to study surface electronic states in various condensed-matter structures ${ }^{57-59}$, is also appropriate for observation of the optically induced topological states discussed above. Since the energy difference between the states and the conduction band is of submeV scale (see Fig. 2), the temperatures around $1 \mathrm{~K}$ are required to observe them experimentally.

\section{Acknowledgments}

The work was partially supported by Horizon2020 RISE project COEXAN, Rannis project 163082-051, Russian Foundation for Basic Research (project 17-0200053), Ministry of Education and Science of Russian Federation (projects 3.4573.2017/6.7, 3.2614.2017/4.6, 14.Y26.31.0015), and the Government of the Russian Federation through the ITMO Fellowship and Professorship Program. O.K. and O.V.K. thank the University of Iceland for hospitality. 
* Electronic address: Oleg.Kibis(c)nstu.ru

1 P. Hänggi, Driven quantum systems, in Quantum Transport and Dissipation edited by T. Dittrich, P. Hänggi, G.L. Ingold, B. Kramer, G. Schön, and W. Zwerger (Wiley, Weinheim, 1998).

2 S. Kohler, J. Lehmann, and P. Hänggi, Driven quantum transport on the nanoscale, Phys. Rep. 406, 379 (2005).

3 M. Bukov, L. D'Alessio, and A. Polkovnikov, Universal High-Frequency Behavior of Periodically Driven Systems: from Dynamical Stabilization to Floquet Engineering, Adv. Phys. 64, 139 (2015).

4 M. Holthaus, Floquet engineering with quasienergy bands of periodically driven optical lattices, J. Phys. B 49, 013001 (2016).

${ }^{5}$ M. Wagner, H. Schneider, D. Stehr, S. Winnerl, A. M. Andrews, S. Schartner, G. Strasser, and M. Helm, Observation of the Intraexciton Autler-Townes Effect in GaAs/AlGaAs Semiconductor Quantum Wells, Phys. Rev. Lett. 105, 167401 (2010).

6 M. Teich, M. Wagner, H. Schneider, and M. Helm, Semiconductor quantum well excitons in strong, narrowband terahertz fields, New J. Phys. 15, 065007 (2013).

7 S. Morina, O. V. Kibis, A. A. Pervishko, and I. A. Shelykh, Transport properties of a two-dimensional electron gas dressed by light, Phys. Rev. B 91, 155312 (2015).

8 A. A. Pervishko, O. V. Kibis, S. Morina, and I. A. Shelykh, Control of spin dynamics in a two-dimensional electron gas by electromagnetic dressing, Phys. Rev. B 92, 205403 (2015).

9 K. Dini, O. V. Kibis, and I. A. Shelykh, Magnetic properties of a two-dimensional electron gas strongly coupled to light, Phys. Rev. B 93, 235411 (2016).

10 O. V. Kibis, O. Kyriienko, and I. A. Shelykh, Persistent current induced by vacuum fluctuations in a quantum ring, Phys. Rev. B 87, 245437 (2013).

11 H. Sigurdsson, O. V. Kibis, and I. A. Shelykh, Optically induced Aharonov-Bohm effect in mesoscopic rings, Phys. Rev. B 90, 235413 (2014).

12 F. K. Joibari, Ya. M. Blanter, and G. E. W. Bauer, Lightinduced spin polarizations in quantum rings, Phys. Rev. B 90, 155301 (2014).

13 K. L. Koshelev, V. Yu. Kachorovskii, and M. Titov, Resonant inverse Faraday effect in nanorings, Phys. Rev. B 92, 235426 (2015).

14 A. Russomanno, Bat-el Friedman, and E. G. Dalla Torre, Spin and topological order in a periodically driven spin chain, Phys. Rev. B 96, 045422 (2017).

15 A. C. Potter and T. Morimoto, Dynamically enriched topological orders in driven two-dimensional systems, Phys. Rev. B 95, 155126 (2017).

16 O. Kyriienko and A. S. Sørensen, Floquet Quantum Simulation with Superconducting Qubits, Phys. Rev. Appl. 9, 064029 (2018).

17 D. M. Kennes, A. de la Torre, A. Ron, D. Hsieh, and A. J. Millis, Floquet Engineering in Quantum Chains, Phys. Rev. Lett. 120, 127601 (2018).

18 T. Oka and H. Aoki, Photovoltaic Hall effect in graphene, Phys. Rev. B 79, 081406 (2009).

19 O. V. Kibis, Metal-insulator transition in graphene induced by circularly polarized photons, Phys. Rev. B 81, 165433 (2010).
${ }^{20}$ O. V. Kibis, O. Kyriienko, and I. A. Shelykh, Band gap in graphene induced by vacuum fluctuations, Phys. Rev. B 84, 195413 (2011).

21 S. V. Syzranov, Ya. I. Rodionov, K. I. Kugel, and F. Nori, Strongly anisotropic Dirac quasiparticles in irradiated graphene, Phys. Rev. B 88, 241112 (2013).

22 M. M. Glazov and S. D. Ganichev, High frequency electric field induced nonlinear effects in graphene, Phys. Rep. 535, 101 (2014).

23 O. V. Kibis, S. Morina, K. Dini, and I. A. Shelykh, Magnetoelectronic properties of graphene dressed by a highfrequency field, Phys. Rev. B 93, 115420 (2016).

${ }^{24}$ K. Kristinsson, O. V. Kibis, S. Morina, and I. A. Shelykh, Control of electronic transport in graphene by electromagnetic dressing, Sci. Rep. 6, 20082 (2016).

25 O. V. Kibis, K. Dini, I. V. Iorsh, and I. A. Shelykh, Alloptical band engineering of gapped Dirac materials, Phys. Rev. B 95, 125401 (2017).

26 A. Iurov, G. Gumbs, and D. Huang, Peculiar electronic states, symmetries and Berry phases in irradiated $\alpha$-T3 materials, arXiv:1806.09172 (2018).

27 M. V. Durnev and S. A. Tarasenko, High-frequency nonlinear transport and photogalvanic effects in two-dimensional topological insulators, arXiv:1901.04181 (2019).

28 M. Z. Hasan, C. L. Kane, Topological insulators, Rev. Mod. Phys. 82, 3045 (2010).

29 C.-K. Chiu, J. C. Y. Teo, A. P. Schnyder, and S. Ryu, Classification of topological quantum matter with symmetries, Rev. Mod. Phys. 88, 035005 (2016).

30 A. Bansil, H. Lin, and T. Das, Topological band theory, Rev. Mod. Phys. 88, 021004 (2016).

31 P. M. Perez-Piskunow, G. Usaj, C. A. Balseiro, and L. E. F. Foa Torres, Floquet chiral edge states in graphene, Phys. Rev. B 89, 121401(R) (2014).

32 G. Usaj, P. M. Perez-Piskunow, L. E. F. Foa Torres, C. A. Balseiro, Irradiated graphene as a tunable Floquet topological insulator Phys. Rev. B 90, 115423 (2014).

33 N. H. Lindner, G. Refael, and V. Galitski, Floquet topological insulator in semiconductor quantum wells, Nat. Phys. 7, 490 (2011).

34 M. Hasan, D. Yudin, I. V. Iorsh, O. Eriksson, I. A. Shelykh, Topological edge-state engineering with highfrequency electromagnetic radiation, Phys. Rev. B 96, 205127 (2017).

35 R. Wang, B. Wang, R. Shen, L. Sheng, and D. Y. Xing, Floquet Weyl semimetal induced by off-resonant light, EPL 105, 17004 (2014).

36 J.-Y. Zou and B.-G. Liu, Floquet Weyl fermions in threedimensional stacked graphene systems irradiated by circularly polarized light, Phys. Rev. B 93, 205435 (2016).

37 X.-X. Zhang, T. T. Ong, and N. Nagaosa, Theory of photoinduced Floquet Weyl semimetal phases, Phys. Rev. B 94, 235137 (2016).

${ }^{38}$ H. Hübener, M. A. Sentef, U. De Giovannini, A. F. Kemper, and A. Rubio, Creating stable Floquet-Weyl semimetals by laser-driving of 3D Dirac materials, Nat. Commun. 8, 13940 (2017).

39 L. Bucciantini, S. Roy, S. Kitamura, and T. Oka, Emergent Weyl nodes and Fermi arcs in a Floquet Weyl semimetal, Phys. Rev. B 96, 041126 (2017).

40 D. Yudin, O. V. Kibis, I. V. Shelykh, Optically tunable 
spin transport on the surface of a topological insulator, New J. Phys. 18, 103014 (2016).

41 A. A. Pervishko, D. Yudin, and I. A. Shelykh, Impact of high-frequency pumping on anomalous finite-size effects in three-dimensional topological insulators, Phys. Rev. B 97, 075420 (2018).

${ }^{42}$ V. K. Kozin, I. V. Iorsh, O. V. Kibis, and I. A. Shelykh, Periodic array of quantum rings strongly coupled to circularly polarized light as a topological insulator, Phys. Rev. B 97, 035416 (2018).

43 X. Dai, T. L. Hughes, X.-L. Qi, Z. Fang, and S.-C. Zhang, Helical edge and surface states in HgTe quantum wells and bulk insulators, Phys. Rev. B 77, 125319 (2008).

44 C. Brüne, C. X. Liu, E. G. Novik, E. M. Hankiewicz, H. Buhmann, Y. L. Chen, X. L. Qi, Z. X. Shen, S. C. Zhang, and L. W. Molenkamp, Quantum Hall Effect from the Topological Surface States of Strained Bulk HgTe, Phys. Rev. Lett, 106, 126803 (2011).

${ }^{45}$ L. Maier, J. B. Oostinga, D. Knott, C. Brüne, P. Virtanen, G. Tkachov, E. M. Hankiewicz, C. Gould, H. Buhmann, and L. W. Molenkamp, Induced Superconductivity in the Three-Dimensional Topological Insulator HgTe, Phys. Rev. Lett. 109, 186806 (2012).

46 B. Yan and S.-C. Zhang, Topological materials, Rep. Prog. Phys. 75, 096501 (2012).

47 J. B. Oostinga, L. Maier, P. Schüffelgen, D. Knott, C. Ames, C. Brüne, G. Tkachov, H. Buhmann, and L. W. Molenkamp, Josephson Supercurrent through the Topological Surface States of Strained Bulk HgTe, Phys. Rev. X 3, 021007 (2013).

48 D. A. Kozlov, Z. D. Kvon, E. B. Olshanetsky, N. N. Mikhailov, S. A. Dvoretsky, and D. Weiss, Transport Properties of a 3D Topological Insulator based on a Strained High-Mobility HgTe Film, Phys. Rev. Lett. 112, 196801 (2014).

49 K.-M. Dantscher, D. A. Kozlov, P. Olbrich, C. Zoth, P. Fal- termeier, M. Lindner, G. V. Budkin, S. A. Tarasenko, V. V. Bel'kov, Z. D. Kvon, N. N. Mikhailov, S. A. Dvoretsky, D. Weiss, B. Jenichen, and S. D. Ganichev, Cyclotronresonance-assisted photocurrents in surface states of a three-dimensional topological insulator based on a strained high-mobility HgTe film, Phys. Rev. B 92, 165314 (2015).

50 J. Ruan, S.-K. Jian, H. Yao, H. Zhang, S.-C. Zhang, and D. Xing, Symmetry-protected ideal Weyl semimetal in HgTeclass materials, Nat. Commun. 7, 11136 (2016).

51 G. L. Bir and G. E. Pikus, Symmetry and Strain-Induced Effects in Semiconductors (Wiley, New York, 1974).

${ }^{52}$ F. Casas, J. A. Oteo, and J. Ros, Floquet theory: exponential perturbative treatment, J. Phys. A 34, 3379 (2001).

53 N. Goldman and J. Dalibard, Periodically Driven Quantum Systems: Effective Hamiltonians and Engineered Gauge Fields, Phys. Rev. X 4, 031027 (2014).

54 A. Eckardt and E. Anisimovas, High-frequency approximation for periodically driven quantum systems from a Floquet-space perspective, New J. Phys. 17, 093039 (2015).

55 S. Adachi, Handbook of physical properties of semiconductors: Vol.3, II-VI compound semiconductors (Kluwer, New York, 2004).

56 M. I. D'yakonov and A. V. Khaetskii, Surface states in a gapless semiconductor, JETP Lett. 33, 110 (1981).

57 Y. Chen, Studies on the electronic structures of threedimensional topological insulators by angle resolved photoemission spectroscopy, Front. Phys. 7, 175 (2012).

58 Y. H. Wang, H. Steinberg, P. Jarillo-Herrero, and N. Gedik, Observation of Floquet-Bloch States on the Surface of a Topological Insulator, Science 342, 453 (2013).

59 F. Mahmood, Ching-Kit Chan, Z. Alpichshev, D. Gardner, Y.Lee, P. A. Lee, and N. Gedik, Selective scattering between Floquet-Bloch and Volkov states in a topological insulator, Nat. Phys. 12, 306 (2016). 